\title{
Targeting the NF-E2-related factor 2 pathway: A novel strategy for glioblastoma (Review)
}

\author{
JIANHONG ZHU, HANDONG WANG, YOUWU FAN, YIXING LIN, \\ LI ZHANG, XIANGJUN JI and MENGLIANG ZHOU
}

\begin{abstract}
Department of Neurosurgery, Jinling Hospital, Medical School of Nanjing University, Nanjing, Jiangsu 210002, P.R. China
\end{abstract}

Received January 23, 2014; Accepted May 26, 2014

DOI: $10.3892 /$ or.2014.3259

\begin{abstract}
Glioblastoma is the most common and malignant subtype among all brain tumors. Nuclear factor erythroid 2-related factor 2 ( Nrf2) is an essential component of cellular defense against a variety of endogenous and exogenous stresses. A marked increase in research over the past few decades focusing on Nrf2 and its role in regulating glioblastoma has revealed the potential value of $\mathrm{Nrf} 2$ in the treatment of glioblastoma. In the present review, we discuss a novel framework of Nrf2 in the regulation of glioblastoma and the mechanisms regarding the downregulation of Nrf2 in treating glioblastoma. The candidate mechanisms include direct and indirect means. Direct mechanisms target tumor molecular pathways in order to overcome resistance to chemotherapy and radiotherapy, to inhibit proliferation, to block invasion and migration, to induce apoptosis, to promote differentiation, to enhance autophagy and to target glioblastoma stem cells. Indirect mechanisms target the reaction between glioblastoma cells and the surrounding microenvironment. Overall, the value of the Nrf2 pathway in glioblastoma provides a promising opportunity for new approaches by which to treat glioblastoma.
\end{abstract}

\section{Contents}

1. Introduction

2. Direct mechanisms

3. Indirect mechanisms

4. Conclusion

Correspondence to: Dr Handong Wang, Department of Neurosurgery, Jinling Hospital, Medical School of Nanjing University, 305 East Zhongshan Road, Nanjing, Jiangsu 210002, P.R. China E-mail: njhdwang@hotmail.com

Key words: glioblastoma, Nrf2, targeting therapy, mechanism

\section{Introduction}

Glioma is one type of brain tumor that arises from glial cells and accounts for over $30 \%$ of all primary central nervous system tumors in the USA (1). Glioblastoma is the most common and malignant subtype of glioma, which is categorized as grade IV according to the classification of the World Health Organization (WHO). The median survival time of glioblastoma patients is approximately 14 months, in spite of aggressive surgery, radiation and chemotherapy (2).

Nuclear factor erythroid 2-related factor 2 (Nrf2) belongs to a subset of basic leucine-zipper (bZip) genes sharing a conserved structural domain (3). It is broadly expressed in tissues and can be activated in response to a range of oxidative and electrophilic stimulation. The activity of $\mathrm{Nrf} 2$ is primarily regulated by its inhibitor Kelch-like ECH-associated protein 1 (Keap1) (4). When uncoupled from the Nrf2/Keap1 complex, Nrf2 is transported into the nucleus and modulates the expression of antioxidant genes through interaction with the antioxidant response element (ARE) (5). An increasing body of literature has revealed alternative mechanisms of Nrf2 activation, including phosphorylation of Nrf2 by various protein kinases, interaction with other protein partners (p21, caveolin-1) and epigenetic factors (microRNA-144, -28 and -200a and promoter methylation) (6).

Recently, Nrf2 has been demonstrated as an important regulator in different types of cancer. A dramatic increase in research focusing on $\mathrm{Nrf} 2$ and the associated mechanisms in the regulation of primary malignant brain tumors such as glioblastoma has been carried out. High expression of Nrf2 in glioblastoma was found to protects it from the killing effects of antitumor therapies, and blocking of Nrf 2 can inhibit glioblastoma. Thus, Nrf2 is a potential new target with which to treat glioblastoma. The mechanisms of the downregulation of Nrf2 in treating glioblastoma contain two main aspects: direct and indirect means. Direct mechanisms target tumor molecular pathways to overcome resistance to chemotherapy and radiotherapy, to inhibit proliferation, to block invasion and migration, to induce apoptosis, to promote differentiation, to enhance autophagy and to target glioblastoma stem cells (GSCs). Indirect mechanisms target the reaction between glioblastoma cells and the surrounding microenvironment, such as the perivascular, hypoxic and immune microenvironments. 
Table I. Direct mechanisms of the downregulation of Nrf2 in the treatment of glioblastoma.

\begin{tabular}{lll}
\hline Mechanism & \multicolumn{1}{c}{ Factors } & Associated molecules \\
\hline Overcoming resistance to chemotherapy & Stress response mechanisms & Phase II detoxifying enzymes \\
& Drug efflux mechanisms & KBCG2 \\
Overcoming resistance to radiotherapy & Endogenous Nrf2 inhibitors & HO-1 \\
Inhibiting proliferation & Downstream molecules & HO-1, GPx2, CXCR3-B \\
& Downstream molecules & EGFR, Ki-67, Kras, PI3K/Akt \\
& Cross-talk & miR-1, miR-200a and miR-206 \\
Blocking invasion and migration & Post-transcriptional regulation & MMP-9 \\
Inducing apoptosis & Matrix metalloproteinases & HO-1 \\
Promoting differentiation & Oxidative stress-related molecules & Bc12, p53, MAPK, NF- $\mathrm{BB}$ \\
& Cross-linking & Notch \\
Enhancing autophagy & Cross-talkg & GST \\
Targeting glioma stem cells & Anti-redox molecules & Keap1, p62, LC3 \\
& P62/SQSTM1 system & UPR \\
& Endoplasmic reticulum stress & MAPK, p53
\end{tabular}

Nrf2, nuclear factor erythroid 2-related factor 2; ABCG2, ATP-binding cassette, subfamily G, member 2; Keap1, Kelch-like ECH-associated protein 1; HO-1, heme oxygenase-1; GPx2, glutathione peroxidase-2; EGFR, epidermal growth factor receptor; MMP-9, matrix metallopro-

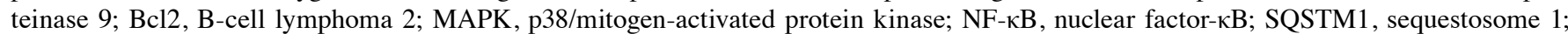
UPR, unfolded protein response; cirDNA, circulating cell-free DNA.

In the present study, we review the function of Nrf2 in the regulation of glioblastoma, and the associated mechanisms concerning the downregulation of Nrf2 in treating glioblastoma.

\section{Direct mechanisms (Table I)}

Overcoming resistance to chemotherapy and radiotherapy. Standard treatment of glioblastoma currently involves chemotherapy and radiotherapy. However, glioblastoma can easily develop resistance to chemotherapy and radiotherapy. It has been found that high expression of Nrf2 decreases the sensitivity of glioblastoma cells to chemotherapy and radiotherapy.

Chemotherapy. There are a variety of tumors that develop strong tolerance to chemotherapy, including glioblastoma (7). Recently, the role of Nrf2 in inducing chemotherapy resistance has been reported in several types of tumors (8). In glioblastoma, Nrf2 expression was found to be increased during drug resistance (8). Temozolomide (TMZ) is an alkylating agent which is commonly used for the treatment of glioblastoma (9-11). TMZ treatment was found to induce Nrf2 activation in the glioblastoma cell line U251 and downregulation of $\mathrm{Nrf} 2$ expression increased TMZ-induced cell death in U251 cells (12). In addition, the silencing of Nrf2 also increased cell necrosis induced by 5-fluorouracil (5-FU), cisplatin, etoposide (13-15), oxaliplatin (16) and doxorubicin (ADM) $(17,18)$. Blocking Nrf2 activation is a potential method for enhancing chemotherapy sensitivity of glioblastoma cells (19).
Nrf2 may induce the chemoresistance of glioblastoma through stress response and a drug efflux mechanism (Fig. 1). The stress response mechanism implies that Nrf2 transcription upregulates endogenous phase II detoxifying enzymes, which may inactivate antitumor drugs by modifying their structures (20). In addition, activation of Nrf2 was also found to contribute to drug efflux pathways (21). ATP-binding cassette, subfamily $\mathrm{G}$, member 2 (ABCG2) plays a crucial role in the efflux of xenobiotics and drugs, and Nrf2-mediated regulation of ABCG2 was found to increase the efflux of antitumor drugs and decrease the effect of chemotherapy (21). However, research suggests that Nrf2 is not an independent molecule in chemoresistance. The possible role of peroxiredoxin1 (Prx1) co-functioning with Nrf2 in chemoresistance has been suggested (22).

Radiotherapy. Radiotherapy is the foundation of therapy following maximal surgical resection of glioblastoma $(23,24)$. However, glioblastoma displays high resistance to radiotherapy (25). Low-dose radiation induces Nrf2 activation reactively (12). The role of $\mathrm{Nrf} 2$ in radioresistance has been investigated. Using a genetically modified method to establish continuous activation of $\mathrm{Nrf} 2$, Nrf2 was found to protect glioblastoma against ionizing radiation toxicity, and Nrf2-inhibited tumor cells showed increased sensitivity to $\gamma$-irradiation (26).

The Nrf2/ARE pathway regulates the radioresistance of glioblastoma by modifying endogenous Nrf2 inhibitor and by upregulating the downstream signal of $\mathrm{Nrf} 2$ (27). Radioresistance may involve the loss-of-function mutations of 


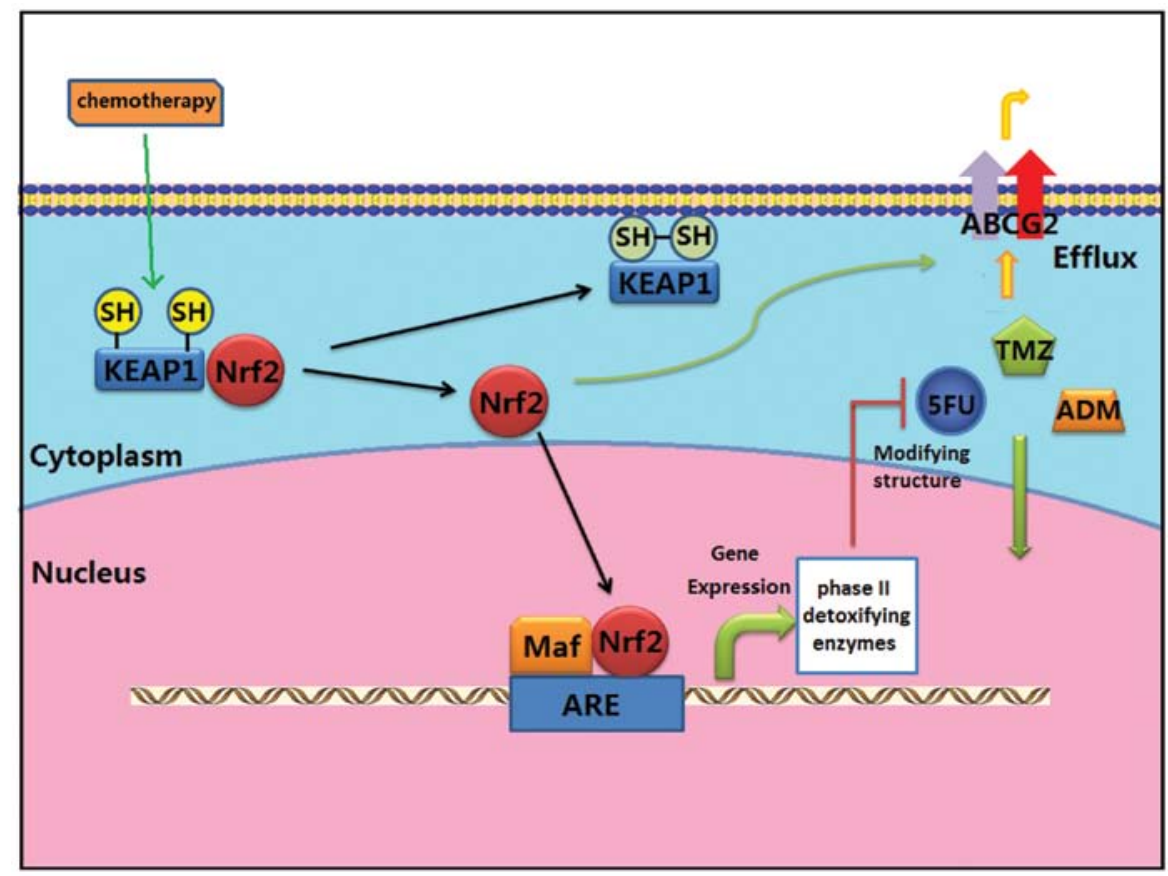

Figure 1. Mechanisms of chemoresistance of glioblastoma induced by Nrf2. Nrf2 is activated by chemotherapy and is transported into the nucleus. Nrf2 binds to the ARE region and promotes the expression of phase II detoxifying enzymes, which inactive antitumor drugs by modifying their structures. In addition, Nrf2 upregulates the expression of ABCG2, increasing the efflux of antitumor drugs. Nrf2, nuclear factor erythroid 2-related factor 2; TMZ, temozolomide; ADM, doxorubicin; 5-FU, 5-fluorouracil; ARE, antioxidant response element; ABCG2, ATP-binding cassette, subfamily G, member 2, Keap1, Kelch-like $\mathrm{ECH}$-associated protein 1.

the Nrf2 inhibitor Keap1, which allows Nrf2 to be continuously transported to the nucleus (28). Other research has demonstrated that Nrf2 induces radioresistance by regulating the function of the major downstream molecule heme oxygenase-1 (HO-1) (29). Downstream activation of Nrf2-ARE-dependent HO-1 was found to be important in the maintenance of resistance to irradiation (12).

Inhibition of proliferation. Glioblastoma cells usually maintain a high rate of proliferation. High expression of $\mathrm{Nrf} 2$ gives glioblastoma an advantage for growth, and knockdown of Nrf2 was found to inhibit the proliferation and growth of human glioblastoma cells $(20,30,31)$.

The candidate mechanisms of Nrf2 in the regulation of proliferation mainly include three means: i) upregulation of downstream molecules of Nrf2; ii) cross-talk with other signaling pathways; iii) and post-transcriptional regulation. Nrf2 can induce the growth of tumor cells by increasing the expression of HO-1, glutathione peroxidase-2 (GPx2) $(32,33)$ and CXCR3-B (34), which are downstream molecules of Nrf2 and are important in the regulation of the growth and proliferation of glioblastoma. The growth rate of cancer cells is inhibited by downregulation of these molecules. Nrf2 is also involved in regulating a variety of other signal transduction pathways. Recently, studies have demonstrated that Nrf 2 can enhance cell proliferation by regulating epidermal growth factor receptor (EGFR), Ki-67, Kras, and phosphoinositide-3-kinase (PI3K)/ Akt pathway, which are necessary for maintaining the proliferation of glioblastoma (35-38). Finally, Nrf2 may improve the accumulation of various proliferation-related proteins by regulating the associated small interfering RNA fraction. Recent studies have identified several microRNAs (miRs) as post-translational targets of $\mathrm{Nrf} 2$ to regulate proliferation. Studies have shown that NADPH and ribose are essential for the cell proliferation in tumors $(39,40)$, and loss of Nrf2 was found to decrease the expression of the redox-sensitive histone deacetylase HDAC4, resulting in increased expression of miR-1, miR-200a and miR-206, which markedly impaired NADPH production and ribose synthesis $(41,42)$.

Blocking of invasion and migration. Glioblastoma can easily invade and migrate to surrounding brain tissue. Nrf2 may facilitate the remodeling of the tumor microenvironment making it advantageous for the autonomic invasion and migration of cancer cells (43). Nrf2 acts as a master switch in these processes by upregulating the expression of various invasion and migration-related proteins (44).

The Nrf2/ARE pathway may regulate glioblastoma invasion and migration through matrix metalloproteinases (MMPs) and oxidative stress-related molecules. MMP activation could improve the degradation of intercellular connections, which enables glioblastoma cells to easily invade and migrate (45). Downregulation of the expression of Nrf2 in the U251 glioblastoma cell line was found to inactivate matrix metalloproteinase-9 (MMP-9) and to decrease the invasion and migration of glioma (44). Oxidative stress is another important mechanism involved in the invasion and migration of glioblastoma. HO-1 is the downstream molecule of $\mathrm{Nrf} 2$, which is important in regulating oxidative stress. Inhibition of HO-1 can weaken the invasive and migratory abilities of glioblastoma $(46,47)$.

However, Thangasamy et al found that the Nrf2 inducer sulforaphane (SFN) can inhibit the expression of tyrosine kinase receptor, recepteur d'origine nantais (RON), which can 


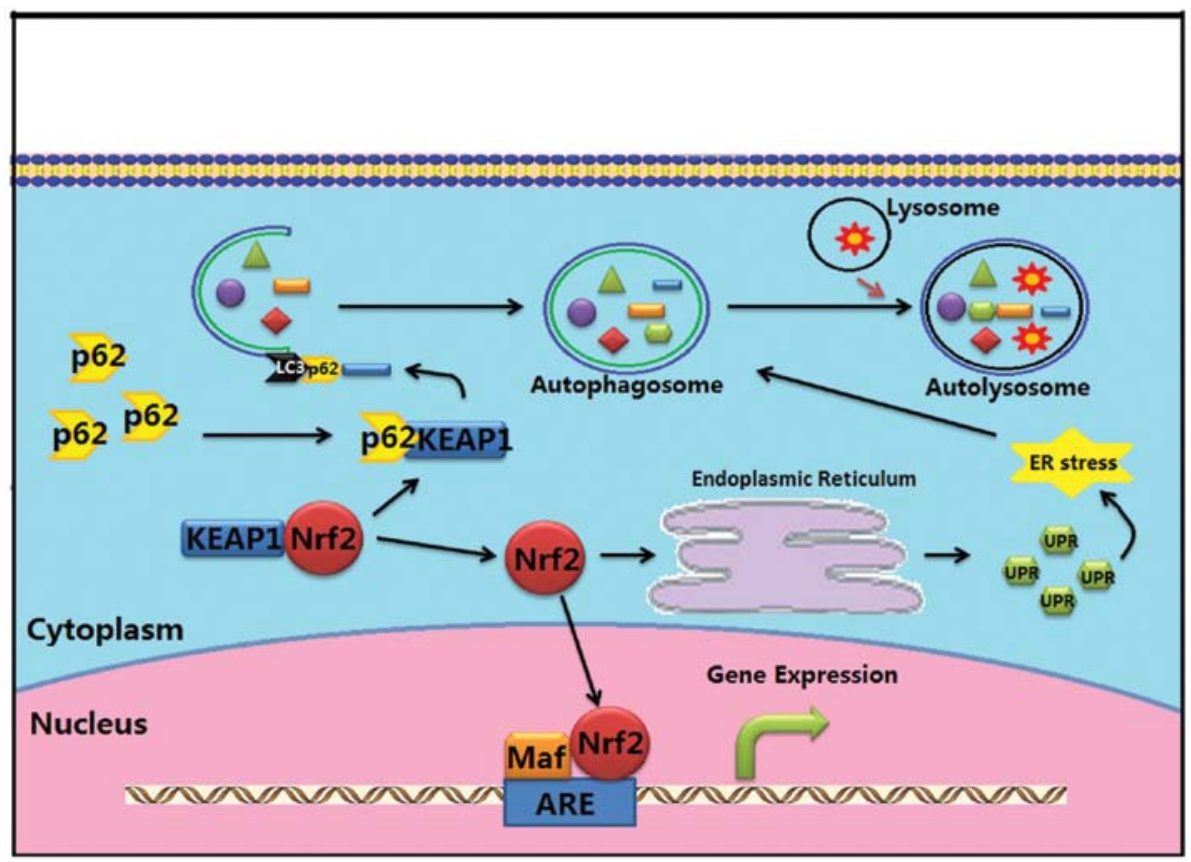

Figure 2. Regulation of autophagy by Nrf2 in glioblastoma. Keap1 uncoupled from the complex with Nrf2, binds to p62, and then interacts with LC3 and is transported to the autophagosome. It is then combined with lysosome to form autolysosome. In addition, Nrf2 can increase the production of the unfolded protein response (UPR) by endoplasmic reticulum (ER), and induce the ER stress to eliminate the UPR by forming autolysosome. Nrf2, nuclear factor erythroid 2-related factor 2; Keap1, Kelch-like ECH-associated protein 1, ARE, antioxidant response element.

mediate the invasion of carcinoma cells (48), indicating that Nrf2 may play a dual role in regulating the invasiveness of tumors.

Induction of apoptosis. In most glioblastoma cells, apoptosis is inhibited (49,50). It has been suggested that Nrf2 can block the apoptotic death of cancer cells (51). Overexpression of Nrf2 was found to significantly diminish apoptosis (52). Inhibition of the Nrf2 transcription factor rendered cancer cells more susceptible to apoptosis (53).

The Nrf2/ARE pathway may regulate apoptosis by cross-linking with the B-cell lymphoma 2 (Bcl2), p53, p38/mitogen-activated protein kinase (MAPK) and nuclear factor- $\kappa \mathrm{B}(\mathrm{NF}-\mathrm{\kappa B})$ pathways. $\mathrm{Bcl} 2$ is an important gene in tumor genesis and in the anti-apoptosis process $(54,55)$. Following increased expression of Nrf2, the expression of caspases 3 was decreased and the apoptosis rate was reduced, accompanied by the upregulated expression of $\mathrm{Bcl}-2 / \mathrm{Bax}$. This indicates that Nrf2 regulates apoptosis through the Bcl2related pathway (56,57). p53 is important due to its anticancer function, and plays an essential role in tumor apoptosis (58). Nrf2 also regulates the tumor-suppressor p53 by influencing the degradation of p53. The Nrf2 downstream molecule NQO1 interacts with $\mathrm{p} 53$ and induces its degradation by the proteasome in a ubiquitin-independent manner (59). In addition, Nrf2 also attenuates the effect of the apoptosis inducer diamide in glioblastoma by upregulating the activity of p38/MAPK and inhibiting the NF-KB pathway $(60,61)$.

Promotion of differentiation. Glioblastoma cells are usually in a poor stage of differentiation and exhibit low maturity (62-64), and differentiation therapy is required as a therapeutic strategy for malignant tumors $(65,66)$. Nrf2 induces the suppression of differentiation by inhibiting a powerful differentiation inducer $1 \alpha, 25$-dihydroxyvitamin D3 $(1,25$ D3) $(67,68)$, suggesting that Nrf2 plays an important role in the cooperative suppression of cancer cell differentiation.

Nrf2 may regulate the differentiation of glioblastoma through cross-talk with the Notch pathway and upregulation of anti-redox molecules. The Notch pathway is important for cell-cell communication, which involves genetic regulatory mechanisms that control the cell differentiation process (69). $\mathrm{Nrf} 2$ adaptive response pathway could directly activate the Notch signal through recruitment of the Notch intracellular domain (NICD) transcriptome and restrain glioblastoma cells in a low state of differentiation (70). In addition, high accumulation of reactive oxygen species (ROS) can induce the differentiation of cells (71). Nrf2 was found to upregulate the anti-redox molecule GST to eliminate ROS and reverse the differentiation induced by ROS $(71,72)$. It has been reported that neuronal differentiation inducer retinoic acid (RA) increased Nrf2 expression reactively $(73,74)$, and downregulation of Nrf2 improves the efficiency of RA in inducing differentiation $(73,74)$.

Enhancement of autophagy. Autophagy is a lysosomal degradation process. Autophagy principally plays an adaptive role to protect organisms against diverse pathological conditions $(75,76)$. Many studies have shed light on the importance of autophagy in glioblastoma (77). Knockdown of Nrf2 was found to regulate the autophagy induced by TMZ in the U251 glioblastoma cell line (78).

Nrf2 may regulate autophagy by altering the P62/SQSTM1 system and endoplasmic reticulum (ER) stress reaction (Fig. 2). 


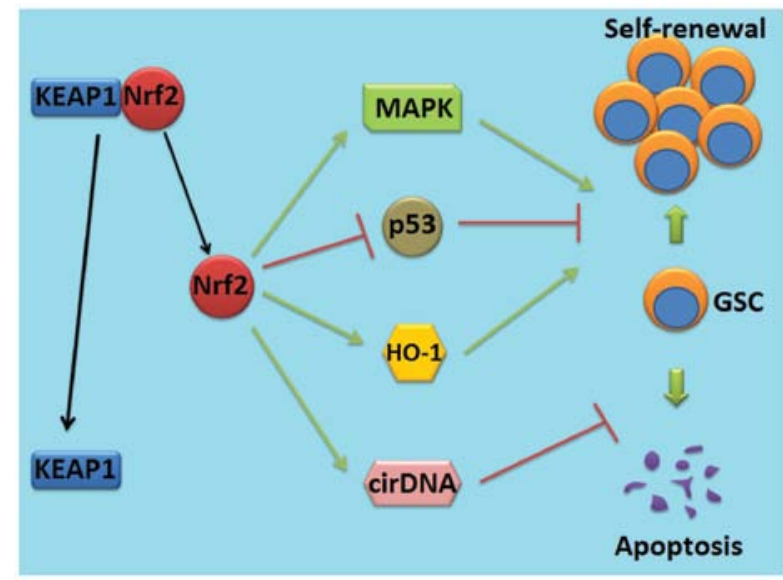

Figure 3. Role of Nrf2 in regulating the function of GSCs in glioblastoma. After uncoupled from the Nrf2/Keap1 complex, Nrf2 increases the expression of MAPK and inhibits p53, along with the Nrf2 downstream compound HO-1, maintaining the self-renewal of GSCs. Nrf2 also regulates the reaction of GSCs to cirDNA, inhibiting the apoptosis of GSCs. Nrf2, nuclear factor erythroid 2-related factor 2; MAPK, mitogen-activated protein kinase; HO-1, heme oxygenase-1; cirDNA, circulating cell-free DNA; GSCs, glioma stem cells, Keap1, Kelch-like ECH-associated protein 1.

The protein of p62, also known as sequestosome 1 (SQSTM1), is one of the adaptors of autophagy. It has been found to play a critical role in the formation of cytoplasmic proteinaceous inclusion. Keap1 uncoupled from the complex with Nrf2 can bind to the autophagy-adaptor protein $\mathrm{p} 62$, and then interacts with LC3 and transports the ubiquitin conjugate to the autophagosome for degradation (79-81). ER stress is a cellular stress response which is activated in response to an accumulation of the unfolded protein response (UPR). High expression of Nrf2 can also induce autophagy by increasing ER stress and by increasing ER-associated degradation (82).

Targeting GSCs. The glioma stem cell (GSC) hypothesis suggests that neoplastic clones are maintained exclusively by a rare fraction of cells with stem cell properties (83). The identification of brain tumor-initiating cells established a new cellular target for more effective therapies (84-86). Over the past decades, Nrf2 was found to be pivotal in the maintenance of the stemness of human GSCs. Knockdown of Nrf2 was found to inhibit the proliferation of GSCs, and significantly reduce the expression of self-renewal-related factors Bmi1, Sox2 and cyclin E (87).

Nrf2 may maintain the stemness of GSCs by cross-linking with MAPK and $\mathrm{p} 53$ pathway, regulating HO-1 and circulating cell-free DNA (cirDNA) (Fig. 3) (88). High expression of Nrf2 can regulate the expression of MAPK and p53 in stem cells, which plays a critical role in the self-renewal of GSCs, indicating that Nrf2 may regulate self-renewal through MAPK and p53 pathway (89). Nrf2 downstream compound HO-1 is important in maintaining the high proliferation of stem cells. The HO-1 inducer cobalt protoporphyrin (CoPP) markedly improved stem cell proliferation (90). Nrf2 also plays an important role in regulating the reaction of stem cells to cirDNA, which is a small fraction of DNA in the plasma and has been found to be important in inhibiting the apoptosis of stem cells. (91).
Table II. Indirect mechanisms of the downregulation of Nrf2 in the treatment of glioblastoma.

\begin{tabular}{ll}
\hline Mechanisms & Factors and associated molecules \\
\hline $\begin{array}{l}\text { Microenvironment } \\
\text { Perivascular }\end{array}$ & HIF-1 $\alpha$, VEGF \\
Hypoxic & HIF-1 $\alpha$, HO-1 \\
Immune & Cytokines: IFN- $\gamma$, IL-4, IL-5, IL-13 \\
& Immune cells: Th, microglia
\end{tabular}

Nrf2, nuclear factor erythroid 2-related factor 2; HIF-1 $\alpha$, hypoxia-inducible factor $\alpha$; VEGF, vascular endothelial growth factor; HO-1, heme oxygenase-1; IFN- $\gamma$, interferon- $\gamma$; Th, T helper cell.

\section{Indirect mechanisms}

The microenvironment is a functional unit enabling complex and dynamic interactions with tumor cells (92). Glioblastoma cells are influenced by non-malignant cells of the tumor microenvironment such as vascular endothelial cells, fibroblasts and immune cells (93). The microenvironment serves as the basis for indirect mechanisms of Nrf2 in the treatment of glioblastoma. Indirect mechanisms include three main aspects of the microenvironment: i) perivascular, ii) hypoxic and iii) immune microenvironment (Table II).

Perivascular microenvironment. Angiogenesis plays a key role in glioblastoma in order to provide energy and maintain the development and progression of glioblastoma. Glioblastoma cells develop a framework to induce the angiogenesis around them $(94,95)$. Recent studies have begun to explore the role of Nrf2 in tumor angiogenesis $(96,97)$. In human glioblastoma cell line U251, knockdown of Nrf2 was found to significantly decrease microvessel density (MVD) and expression of small vessel marker CD31 (38).

Nrf2 may regulate angiogenesis through hypoxia-inducible factor $1 \alpha$ (HIF-1 $\alpha$ ) and vascular endothelial growth factors (VEGFs). As a main downstream molecule of Nrf2, HIF-1 $\alpha$ is one of the master regulators that orchestrate cellular responses to hypoxia. Activation of HIF-1 $\alpha$ can lead to the activation of numerous perivascular compounds, such as angiopoietin, endothelin-1, inducible nitric oxide synthase (iNOS), adrenomedullin and erythropoietin. Blocking HIF-1 $\alpha$ can inhibit the angiogenesis effect of Nrf2 (98). Another important inducer of vessels is VEGF. Nrf2 elevates VEGF expression and improves the growth of the vascular endothelia in tumors. Through a positive feedback loop, VEGF can also activate Nrf2 in an ERK1/2-dependent manner and induce the production of antioxidative enzymes (99). Anti-angiogenesis effects of Nrf2 knockdown were documented in chick chorioallantoic membrane assays and endothelial tube formation assays (100).

Hypoxic microenvironment. Hypoxia and tumor genesis are closely related (101). Glioblastoma has extensive areas of hypoxia and displays high tolerance to a low concentration of oxygen $(102,103)$. Nrf2 has been identified as a regulator of 
several genes involved in the hypoxic defense response, such as HIF-1 $\alpha$ (104). In human glioblastoma, high expression of Nrf2 was significantly correlated with high tolerance to a low concentration of oxygen, less tumor necrosis on MRI and lower 1-year survival of patients (105).

It is believed that Nrf2 regulates the hypoxia resistance by HIF- $1 \alpha$ and HO-1. HIF- $1 \alpha$ is a downstream molecule of Nrf2 and is one of the master regulators of hypoxia (98). In a $\mathrm{CoCl}_{2}$-induced hypoxia model, blockage of Nrf2 suppressed the expression of HIF-1 $\alpha$, and suppressed the migration and invasion of tumors in a hypoxic microenvironment (106). HO-1 is another important molecule for resistance to hypoxia. In a 6-hydroxydopamine (6-OHDA)-induced hypoxic model, Nrf2 activation induced upregulation of HO-1, and mediated the cellular adaptive survival response to a hypoxic microenvironment (107).

Immune microenvironment. Glioblastoma can escape from tumor immunosurveillance and inactivate the reaction between tumors and immune cells. The immune microenvironment surounding glioblastoma plays an important role in these processes (108). In addition, Nrf2 was also found to be a critical regulator of the immune reaction (109).

The Nrf2/ARE pathway may regulate tumor immunosurveillance through regulation of the secretion of cytokines and the function of immune cells. Nrf2 regulates the secretion of many types of cytokines. Activation of Nrf2 was found to suppress the production of interferon- $\gamma($ IFN- $\gamma$ ), while inducing the production of T helper cells 2 (Th2), cytokines IL-4, IL-5, and IL-13 (110). Nrf2 also regulates the function of immune cells. In glioblastoma, T helper cells (Th) play an important role in the adaptive immune system. Th helps the activation of other immune cells by releasing T cell cytokines. Nrf2 is a regulator of Th and activates CD4(+) T cells from differentiating towards $\mathrm{Th} 2$, representing a novel regulatory mechanism in CD4(+) T cells (111). Microglia act as the main form of active immune defense in the central nervous system (CNS). Nrf2 also mediates immunoresistance by modifying the function of microglia. Activation of the Nrf2/HO-1 pathway was found to suppress BV2 microglial cells and immunology in the brain (112). Upregulation of Nrf2 suppressed innate immune microglial cells in the CNS. Various small activators of Nrf2/ HO-1 such as carnosol, supercurcumin and dimethyl fumarate are effective modulators of microglial-related immune responses (112).

\section{Conclusion}

In the past decades, a marked increasing in research has been carried out focusing on Nrf2 and its role in regulating glioblastoma and the possibilities of the downregulation of $\mathrm{Nrf} 2$ for treating glioblastoma. Nrf2 plays an extensively role in the regulation of glioblastoma; hence, downregulation of Nrf2 can interfere with a variety of behaviors of glioblastoma and actions of the microenvironment surrounding glioblastoma. Thus Nrf2 has promising value as a therapeutic target for glioblastoma. However, Nrf2 downregulation in most studies was obtained through RNA interference or knockdown technology, rather than pharmaceutical compounds, making targeted Nrf2 therapy somewhat difficult and less appealing at this time from a translational perspective. Recently, biochemists have identified the small molecule, ochratoxin $\mathrm{A}$, as an inhibitor of Nrf2 (113). Although it is a toxin produced by Aspergillus ochraceus, the single compound is a potential new strategy with which to inhibit Nrf2 in glioblastoma. For these reasons, future studies should focus on regulatory methods of $\mathrm{Nrf} 2$, which can be easily translated to the clinical setting and be used safely.

\section{Acknowledgements}

This study was supported by grants from the National Natural Science Foundation of China (nos. 81070974 and 81271377), the Jiangsu Provincial Key Subject (no. X4200722), and Jinling Hospital (no. 2010Q017).

\section{References}

1. Binello E and Germano IM: Targeting glioma stem cells: a novel framework for brain tumors. Cancer Sci 102: 1958-1966, 2011.

2. Van Meir EG, Hadjipanayis CG, Norden AD, et al: Exciting new advances in neuro-oncology: the avenue to a cure for malignant glioma. CA Cancer J Clin 60: 166-193, 2010.

3. Kensler TW, Wakabayashi $\mathrm{N}$ and Biswal S: Cell survival responses to environmental stresses via the Keap1-Nrf2-ARE pathway. Annu Rev Pharmacol Toxicol 47: 89-116, 2007.

4. Reuter S, Gupta SC, Chaturvedi MM and Aggarwal BB: Oxidative stress, inflammation, and cancer: how are they linked? Free Radic Biol Med 49: 1603-1616, 2010.

5. Li H, Wang F, Zhang L, et al: Modulation of Nrf2 expression alters high glucose-induced oxidative stress and antioxidant gene expression in mouse mesangial cells. Cell Signal 23: 1625-1632, 2011.

6. Bryan HK, Olayanju A, Goldring CE and Park BK: The Nrf2 cell defence pathway: Keap1-dependent and -independent mechanisms of regulation. Biochem Pharmacol 85: 705-717, 2013.

7. Livingston DM and Silver DP: Cancer: crossing over to drug resistance. Nature 451: 1066-1067, 2008.

8. Chen Q, Li W, Wan Y, et al: Amplified in breast cancer 1 enhances human cholangiocarcinoma growth and chemoresistance by simultaneous activation of Akt and Nrf2 pathways. Hepatology 55: 1820-1829, 2012.

9. Stupp R, Mason WP, van den Bent MJ, et al: Radiotherapy plus concomitant and adjuvant temozolomide for glioblastoma. N Engl J Med 352: 987-996, 2005.

10. Newlands ES, Stevens MF, Wedge SR, et al: Temozolomide: a review of its discovery, chemical properties, pre-clinical development and clinical trials. Cancer Treat Rev 23: 35-61, 1997.

11. Friedman HS, Kerby $\mathrm{T}$ and Calvert H: Temozolomide and treatment of malignant glioma. Clin Cancer Res 6: 2585-2597, 2000.

12. Cong ZX, Wang HD, Zhou Y, et al: Temozolomide and irradiation combined treatment-induced Nrf 2 activation increases chemoradiation sensitivity in human glioblastoma cells. J Neurooncol 116: 41-48, 2014.

13. Hu XF, Yao J, Gao SG, et al: Nrf2 overexpression predicts prognosis and 5-fu resistance in gastric cancer. Asian Pac J Cancer Prev 14: 5231-5235, 2013.

14. Jiang T, Chen N, Zhao F, et al: High levels of Nrf2 determine chemoresistance in type II endometrial cancer. Cancer Res 70: 5486-5496, 2010.

15. Wang XJ, Sun Z, Villeneuve NF, et al: Nrf2 enhances resistance of cancer cells to chemotherapeutic drugs, the dark side of $\mathrm{Nrf} 2$. Carcinogenesis 29: 1235-1243, 2008.

16. Chen CC, Chu CB, Liu KJ, et al: Gene expression profiling for analysis acquired oxaliplatin resistant factors in human gastric carcinoma TSGH-S3 cells: the role of IL-6 signaling and Nrf2/AKR1C axis identification. Biochem Pharmacol 86: 872-887, 2013.

17. Gao AM, Ke ZP, Shi F, et al: Chrysin enhances sensitivity of BEL-7402/ADM cells to doxorubicin by suppressing PI3K/Akt/ Nrf2 and ERK/Nrf2 pathway. Chem Biol Interact 206: 100-108, 2013. 
18. Gao AM, Ke ZP, Wang JN, et al: Apigenin sensitizes doxorubicin-resistant hepatocellular carcinoma BEL-7402/ADM cells to doxorubicin via inhibiting PI3K/Akt/Nrf2 pathway. Carcinogenesis 34: 1806-1814, 2013.

19. Kim WD, Kim YW, Cho IJ, et al: E-cadherin inhibits nuclear accumulation of Nrf2: implications for chemoresistance of cancer cells. J Cell Sci 125: 1284-1295, 2012.

20. Lau A, Villeneuve NF, Sun Z, et al: Dual roles of Nrf2 in cancer. Pharmacol Res 58: 262-270, 2008.

21. Singh A, Wu H, Zhang P, et al: Expression of ABCG2 (BCRP) is regulated by Nrf2 in cancer cells that confers side population and chemoresistance phenotype. Mol Cancer Ther 9: 2365-2376, 2010.

22. Kim JH, Bogner PN, Ramnath N, et al: Elevated peroxiredoxin 1, but not NF-E2-related factor 2, is an independent prognostic factor for disease recurrence and reduced survival in stage I non-small cell lung cancer. Clin Cancer Res 13: 3875-3882, 2007.

23. Alexander BM, Ligon KL and Wen PY: Enhancing radiation therapy for patients with glioblastoma. Expert Rev Anticancer Ther 13: 569-581, 2013.

24. Caruso C, Carcaterra M and Donato V: Role of radiotherapy for high grade gliomas management. J Neurosurg Sci 57: 163-169, 2013.

25. Frosina G: DNA repair and resistance of gliomas to chemotherapy and radiotherapy. Mol Cancer Res 7: 989-999, 2009.

26. Singh A, Bodas M, Wakabayashi N, et al: Gain of Nrf2 function in non-small-cell lung cancer cells confers radioresistance. Antioxid Redox Signal 13: 1627-1637, 2010.

27. Sharma PK and Varshney R: 2-Deoxy-D-glucose and 6-aminonicotinamide-mediated $\mathrm{Nrf} 2$ down regulation leads to radiosensitization of malignant cells via abrogation of GSH-mediated defense. Free Radic Res 46: 1446-1457, 2012.

28. Zhang P, Singh A, Yegnasubramanian S, et al: Loss of Kelch-like $\mathrm{ECH}$-associated protein 1 function in prostate cancer cells causes chemoresistance and radioresistance and promotes tumor growth. Mol Cancer Ther 9: 336-346, 2010.

29. Na HK and Surh YJ: Oncogenic potential of Nrf2 and its principal target protein heme oxygenase-1. Free Radic Biol Med 67: 353-365, 2014

30. DeNicola GM, Karreth FA, Humpton TJ, et al: Oncogene-induced Nrf2 transcription promotes ROS detoxification and tumorigenesis. Nature 475: 106-109, 2011.

31. Taguchi K, Motohashi $\mathrm{H}$ and Yamamoto M: Molecular mechanisms of the Keap1-Nrf2 pathway in stress response and cancer evolution. Genes Cells 16: 123-140, 2011.

32. Brigelius-Flohé R, Müller M, Lippmann D and Kipp AP: The yin and yang of nrf2-regulated selenoproteins in carcinogenesis. Int J Cell Biol 2012: 486147, 2012.

33. Lu SC: Regulation of glutathione synthesis. Mol Aspects Med 30: 42-59, 2009.

34. Balan M and Pal S: A novel CXCR3-B chemokine receptorinduced growth-inhibitory signal in cancer cells is mediated through the regulation of Bach-1 protein and $\mathrm{Nrf} 2$ protein nuclear translocation. J Biol Chem 289: 3126-3137, 2014.

35. Yamadori T, Ishii Y, Homma S, et al: Molecular mechanisms for the regulation of Nrf2-mediated cell proliferation in non-smallcell lung cancers. Oncogene 31: 4768-4777, 2012.

36. Zou W, Chen C, Zhong Y, et al: PI3K/Akt pathway mediates Nrf2/ARE activation in human L02 hepatocytes exposed to lowconcentration HBCDs. Environ Sci Technol 47: 12434-12440, 2013.

37. Kong B, Qia C, Erkan M, et al: Overview on how oncogenic Kras promotes pancreatic carcinogenesis by inducing low intracellular ROS levels. Front Physiol 4: 246, 2013.

38. Ji XJ, Chen SH, Zhu L, et al: Knockdown of NF-E2-related factor 2 inhibits the proliferation and growth of U251MG human glioma cells in a mouse xenograft model. Oncol Rep 30: 157-164, 2013.

39. Crosas-Molist E, Bertran E, Sancho P, et al: The NADPH oxidase NOX4 inhibits hepatocyte proliferation and liver cancer progression. Free Radic Biol Med 69: 338-347, 2014.

40. Wang Z, Li Y, Lv S and Tian Y: Inhibition of proliferation and invasiveness of ovarian cancer $\mathrm{C} 13^{*}$ cells by a poly(ADP-ribose) polymerase inhibitor and the role of nuclear factor- $\mathrm{kB}$. J Int Med Res 41: 1577-1585, 2013.

41. Singh A, Happel C, Manna SK, et al: Transcription factor NRF2 regulates miR-1 and miR-206 to drive tumorigenesis. J Clin Invest 123: 2921-2934, 2013.
42. Petrelli A, Perra A, Cora D, et al: MicroRNA/gene profiling unveils early molecular changes and nuclear factor erythroid related factor 2 (NRF2) activation in a rat model recapitulating human hepatocellular carcinoma (HCC). Hepatology 59: 228-241, 2014.

43. Rachakonda G, Sekhar KR, Jowhar D, et al: Increased cell migration and plasticity in Nrf2-deficient cancer cell lines. Oncogene 29: 3703-3714, 2010.

44. Pan H, Wang H, Zhu L, et al: The role of Nrf2 in migration and invasion of human glioma cell U251. World Neurosurg 80: 363-370, 2013.

45. Deryugina EI, Bourdon MA, Luo GX, et al: Matrix metalloproteinase-2 activation modulates glioma cell migration. J Cell Sci 110: 2473-2482, 1997.

46. Gan FF, Ling H, Ang X, et al: A novel shogaol analog suppresses cancer cell invasion and inflammation, and displays cytoprotective effects through modulation of NF- $\kappa \mathrm{B}$ and Nrf2-Keap1 signaling pathways. Toxicol Appl Pharmacol 272: 852-862, 2013.

47. Zhang L, Wang N, Zhou S, et al: Propofol induces proliferation and invasion of gallbladder cancer cells through activation of Nrf2. J Exp Clin Cancer Res 31: 66, 2012.

48. Thangasamy A, Rogge J, Krishnegowda NK, et al: Novel function of transcription factor $\mathrm{Nrf} 2$ as an inhibitor of RON tyrosine kinase receptor-mediated cancer cell invasion. J Biol Chem 286: 32115-32122, 2011.

49. Evan GI and Vousden KH: Proliferation, cell cycle and apoptosis in cancer. Nature 411: 342-348, 2001.

50. Johnstone RW, Ruefli AA and Lowe SW: Apoptosis: a link between cancer genetics and chemotherapy. Cell 108: 153-164, 2002.

51. Bat-Chen W, Golan T, Peri I, et al: Allicin purified from fresh garlic cloves induces apoptosis in colon cancer cells via $\mathrm{Nrf} 2$. Nutr Cancer 62: 947-957, 2010.

52. Chen X, Liu J and Chen SY: Over-expression of Nrf2 diminishes ethanol-induced oxidative stress and apoptosis in neural crest cells by inducing an antioxidant response. Reprod Toxicol 42: 102-109, 2013.

53. Arlt A, Sebens S, Krebs S, et al: Inhibition of the Nrf2 transcription factor by the alkaloid trigonelline renders pancreatic cancer cells more susceptible to apoptosis through decreased proteasomal gene expression and proteasome activity. Oncogene 32: 4825-4835, 2013.

54. Liston P, Fong WG and Korneluk RG: The inhibitors of apoptosis: there is more to life than Bcl2. Oncogene 22: 8568-8580, 2003.

55. Thomadaki $\mathrm{H}$ and Scorilas A: BCL2 family of apoptosis-related genes: functions and clinical implications in cancer. Crit Rev Clin Lab Sci 43: 1-67, 2006.

56. Heasman SA, Zaitseva L, Bowles KM, et al: Protection of acute myeloid leukaemia cells from apoptosis induced by front-line chemotherapeutics is mediated by haem oxygenase-1. Oncotarget 2: 658-668, 2011.

57. Niture SK and Jaiswal AK: INrf2 (Keap1) targets Bcl-2 degradation and controls cellular apoptosis. Cell Death Differ 18: 439-451, 2011.

58. Attardi LD: The role of p53-mediated apoptosis as a crucial anti-tumor response to genomic instability: lessons from mouse models. Mutat Res 569: 145-157, 2005.

59. Rotblat B, Melino G and Knight RA: NRF2 and p53: Januses in cancer? Oncotarget 3: 1272-1283, 2012.

60. Filomeni G, Piccirillo S, Rotilio G and Ciriolo MR: p38 $8^{\mathrm{MAPK}}$ and ERK1/2 dictate cell death/survival response to different pro-oxidant stimuli via p53 and Nrf2 in neuroblastoma cells SH-SY5Y. Biochem Pharmacol 83: 1349-1357, 2012.

61. Lee YM, Auh QS, Lee DW, et al: Involvement of Nrf2-mediated upregulation of heme oxygenase-1 in mollugin-induced growth inhibition and apoptosis in human oral cancer cells. Biomed Res Int 2013: 210604, 2013.

62. Sell S: Stem cell origin of cancer and differentiation therapy. Crit Rev Oncol Hematol 51: 1-28, 2004

63. Bollag W and Holdener EE: Retinoids in cancer prevention and therapy. Ann Oncol 3: 513-526, 1992.

64. Clarke N, Germain P, Altucci L and Gronemeyer H: Retinoids: potential in cancer prevention and therapy. Expert Rev Mol Med 6: 1-23, 2004.

65. Hansen LA, Sigman CC, Andreola F, et al: Retinoids in chemoprevention and differentiation therapy. Carcinogenesis 21: $1271-1279,2000$. 
66. Leszczyniecka M, Roberts T, Dent P, et al: Differentiation therapy of human cancer: basic science and clinical applications. Pharmacol Ther 90: 105-156, 2001.

67. Bobilev I, Novik V, Levi I, et al: The Nrf2 transcription factor is a positive regulator of myeloid differentiation of acute myeloid leukemia cells. Cancer Biol Ther 11: 317-329, 2011.

68. Li K, Zhong C, Wang B, et al: Nrf2 expression participates in growth and differentiation of endometrial carcinoma cells in vitro and in vivo. J Mol Histol 45: 161-167, 2014.

69. Capaccione KM and Pine SR: The Notch signaling pathway as a mediator of tumor survival. Carcinogenesis 34: 1420-1430, 2013.

70. Wakabayashi N, Skoko JJ, Chartoumpekis DV, et al: Notch-Nrf2 axis: regulation of $N r f 2$ gene expression and cytoprotection by Notch signaling. Mol Cell Biol 34: 653-663, 2014.

71. Kanzaki H, Shinohara F, Kajiya M and Kodama T: The Keap1/Nrf2 protein axis plays a role in osteoclast differentiation by regulating intracellular reactive oxygen species signaling. J Biol Chem 288 : 23009-23020, 2013.

72. Jayakumar S, Kunwar A, Sandur SK, et al: Differential response of DU145 and PC3 prostate cancer cells to ionizing radiation: role of reactive oxygen species, GSH and $\mathrm{Nrf} 2$ in radiosensitivity. Biochim Biophys Acta 1840: 485-494, 2014.

73. Wang XJ, Hayes JD, Henderson CJ and Wolf CR: Identification of retinoic acid as an inhibitor of transcription factor $\mathrm{Nrf} 2$ through activation of retinoic acid receptor alpha. Proc Natl Acad Sci USA 104: 19589-19594, 2007.

74. Tan KP, Kosuge K, Yang M and Ito S: NRF2 as a determinant of cellular resistance in retinoic acid cytotoxicity. Free Radic Biol Med 45: 1663-1673, 2008

75. Levine B and Kroemer G: Autophagy in the pathogenesis of disease. Cell 132: 27-42, 2008.

76. Mizushima N, Levine B, Cuervo AM and Klionsky DJ: Autophagy fights disease through cellular self-digestion. Nature 451: 1069-1075, 2008.

77. Kondo Y, Kanzawa T, Sawaya R and Kondo S: The role of autophagy in cancer development and response to therapy. Nat Rev Cancer 5: 726-734, 2005.

78. Zhou Y, Wang HD, Zhu L, et al: Knockdown of Nrf2 enhances autophagy induced by temozolomide in U251 human glioma cell line. Oncol Rep 29: 394-400, 2013.

79. Fan W, Tang Z, Chen D, et al: Keap1 facilitates p62-mediated ubiquitin aggregate clearance via autophagy. Autophagy 6 : 614-621, 2010

80. Kwon J, Han E, Bui CB, et al: Assurance of mitochondrial integrity and mammalian longevity by the p62-Keap1-Nrf2-Nqo1 cascade. EMBO Rep 13: 150-156, 2012.

81. Inami Y, Waguri S, Sakamoto A, et al: Persistent activation of Nrf2 through p62 in hepatocellular carcinoma cells. J Cell Biol 193: 275-284, 2011.

82. Digaleh H, Kiaei M and Khodagholi F: Nrf2 and Nrf1 signaling and ER stress crosstalk: implication for proteasomal degradation and autophagy. Cell Mol Life Sci 70: 4681-4694, 2013.

83. Reya T, Morrison SJ, Clarke MF and Weissman IL: Stem cells, cancer, and cancer stem cells. Nature 414: 105-111, 2001.

84. Singh SK, Hawkins C, Clarke ID, et al: Identification of human brain tumour initiating cells. Nature 432: 396-401, 2004.

85. Singh SK, Clarke ID, Terasaki M, et al: Identification of a cancer stem cell in human brain tumors. Cancer Res 63: 5821-5828, 2003.

86. Bao S, Wu Q, McLendon RE, et al: Glioma stem cells promote radioresistance by preferential activation of the DNA damage response. Nature 444: 756-760, 2006.

87. Zhu J, Wang H, Sun Q, et al: Nrf2 is required to maintain the self-renewal of glioma stem cells. BMC Cancer 13: 380, 2013.

88. Tsai JJ, Dudakov JA, Takahashi K, et al: Nrf2 regulates haematopoietic stem cell function. Nat Cell Biol 15: 309-316, 2013.

89. Wang K, Zhang T, Dong Q, et al: Redox homeostasis: the linchpin in stem cell self-renewal and differentiation. Cell Death Dis 4: e537, 2013.

90. Cai C, Teng L, Vu D, et al: The heme oxygenase 1 inducer (CoPP) protects human cardiac stem cells against apoptosis through activation of the extracellular signal-regulated kinase (ERK)/NRF2 signaling pathway and cytokine release. J Biol Chem 287: 33720-33732, 2012.

91. Loseva P, Kostyuk S, Malinovskaya E, et al: Extracellular DNA oxidation stimulates activation of NRF2 and reduces the production of ROS in human mesenchymal stem cells. Expert Opin Biol Ther 12 (Suppl 1): S85-S97, 2012.
92. Hoelzinger DB, Demuth T and Berens ME: Autocrine factors that sustain glioma invasion and paracrine biology in the brain microenvironment. J Natl Cancer Inst 99: 1583-1593, 2007.

93. Joyce JA and Pollard JW: Microenvironmental regulation of metastasis. Nat Rev Cancer 9: 239-252, 2009.

94. Bergers $\mathrm{G}$ and Benjamin LE: Tumorigenesis and the angiogenic switch. Nat Rev Cancer 3: 401-410, 2003.

95. Folkins C, Man S, Xu P, et al: Anticancer therapies combining antiangiogenic and tumor cell cytotoxic effects reduce the tumor stem-like cell fraction in glioma xenograft tumors. Cancer Res 67: 3560-3564, 2007.

96. Zhou S, Ye W, Zhang M and Liang J: The effects of nrf2 on tumor angiogenesis: a review of the possible mechanisms of action. Crit Rev Eukaryot Gene Expr 22: 149-160, 2012.

97. Ashino T, Yamamoto M, Yoshida T and Numazawa S: Redoxsensitive transcription factor Nrf2 regulates vascular smooth muscle cell migration and neointimal hyperplasia. Arterioscler Thromb Vasc Biol 33: 760-768, 2013.

98. Kaur B, Khwaja FW, Severson EA, et al: Hypoxia and the hypoxia-inducible-factor pathway in glioma growth and angiogenesis. Neuro Oncol 7: 134-153, 2005.

99. Kweider N, Fragoulis A, Rosen C, et al: Interplay between vascular endothelial growth factor (VEGF) and nuclear factor erythroid 2-related factor-2 (Nrf2): implications for preeclampsia. J Biol Chem 286: 42863-42872, 2011

100. Kim TH, Hur EG, Kang SJ, et al: NRF2 blockade suppresses colon tumor angiogenesis by inhibiting hypoxia-induced activation of HIF-1 $\alpha$. Cancer Res 71: 2260-2275, 2011.

101. Ji RC: Hypoxia and lymphangiogenesis in tumor microenvironment and metastasis. Cancer Lett 346: 6-16, 2014

102. Emara M and Allalunis-Turner J: Effect of hypoxia on angiogenesis related factors in glioblastoma cells. Oncol Rep 31: 1947-1953, 2014

103. Marampon F, Gravina GL, Zani BM, et al: Hypoxia sustains glioblastoma radioresistance through ERKs/DNA-PKcs/HIF-1a functional interplay. Int J Oncol 44: 2121-2131, 2014.

104. Ji X, Wang H, Zhu J, et al: Knockdown of Nrf2 suppresses glioblastoma angiogenesis by inhibiting hypoxia-induced activation of HIF-1 $\alpha$. Int J Cancer 135: 574-584, 2014.

105. Ji X, Wang H, Zhu J, et al: Correlation of Nrf2 and HIF-1a in glioblastoma and their relationships to clinicopathologic features and survival. Neurol Res 35: 1044-1050, 2013.

106. Shen H, Yang Y, Xia S, et al: Blockage of Nrf2 suppresses the migration and invasion of esophageal squamous cell carcinoma cells in hypoxic microenvironment. Dis Esophagus: Sep 13, 2013 (Epub ahead of print).

107. Lee C, Park GH and Jang JH: Cellular antioxidant adaptive survival response to 6-hydroxydopamine-induced nitrosative cell death in C6 glioma cells. Toxicology 283: 118-128, 2011.

108. Meisen WH and Kaur B: How can we trick the immune system into overcoming the detrimental effects of oncolytic viral therapy to treat glioblastoma? Expert Rev Neurother 13: 341-343, 2013.

109. Al-Huseini LM, Aw Yeang HX, Sethu S, et al: Nuclear factorerythroid 2 (NF-E2) p45-related factor-2 (Nrf2) modulates dendritic cell immune function through regulation of p38 MAPK-cAMP-responsive element binding protein/activating transcription factor 1 signaling. J Biol Chem 288: 22281-22288, 2013.

110. Thimmulappa RK, Lee H, Rangasamy T, et al: Nrf2 is a critical regulator of the innate immune response and survival during experimental sepsis. J Clin Invest 116: 984-995, 2006.

111. Rockwell CE, Zhang M, Fields PE and Klaassen CD: Th2 skewing by activation of $\mathrm{Nrf} 2$ in $\mathrm{CD}^{+}{ }^{+} \mathrm{T}$ cells. J Immunol 188 1630-1637, 2012.

112. Foresti R, Bains SK, Pitchumony TS, et al: Small molecule activators of the Nrf2-HO-1 antioxidant axis modulate heme metabolism and inflammation in BV2 microglia cells. Pharmacol Res 76: 132-148, 2013.

113. Limonciel A and Jennings P: A review of the evidence that ochratoxin A is an Nrf2 inhibitor: implications for nephrotoxicity and renal carcinogenicity. Toxins 6: 371-379, 2014. 